\title{
Increased rates of wound complications with locking plates in distal fibular
}

fractures

T. Schepers ${ }^{1}$, E.M.M. van Lieshout ${ }^{2}$, M.R. de Vries ${ }^{1}$, M. Van der Elst ${ }^{1}$

\section{Schepers@rdgg.nl}

E.vanlieshout@erasmusmc.nl

VriesM@rdgg.nl

Elst@rdgg.nl

${ }^{1}$ Department of Surgery and Traumatology, Reinier de Graaf Groep Delft, The Netherlands

${ }^{2}$ Department of Surgery-Traumatology, Erasmus MC, University Medical Center Rotterdam, Rotterdam, The Netherlands

Corresponding author:

T. Schepers, MD PhD

Department of Surgery and Traumatology

Reinier de Graaf Groep Delft

PO Box 5011

2600 GA Delft

The Netherlands

Tel: $+31-15-2603060$

Fax: +31-15-2603599

$\underline{\text { Schepers@ @rdgg.nl }}$ 
Keywords: ankle fracture, operative treatment, complications, locking plate 


\section{Abstract}

Introduction: There is a growing use of locking compression plates in fracture surgery. The current study was undertaken to investigate the wound complication rates of locking versus non-locking plates in distal fibular fractures.

Patients and Methods: During a six year study period all consecutive, closed distal fibular fractures treated with a locking or non-locking plate were included and retrospectively analysed for complication related to the fibula.

Results: A total of 165 patients received a one-third tubular plate and 40 patients were treated with a locking plate. The two groups were comparable with respect to patient characteristics (age, gender, smokers, and diabetics), injury characteristics (affected side, fracture -dislocations, number of fractured malleoli, and classification), and operation characteristics (surgical delay and duration, use of a tourniquet, and plate length). The wound complication rate was 5.5 percent in the conventional plating group, and 17.5 percent in the locking plate group $(\mathrm{p}=0.019)$. This difference was largely due to an increase in major complications, for which removal of the plate was necessary $(\mathrm{p}=$ $0.008)$.

Conclusion: there is a significant increase in wound complications in distal fibular fractures treated with a locking compression plate. In light of the current study we would caution against the application of the currently used locking compression plates in the treatment of distal fibular fractures. 


\section{Introduction}

During the last decade locking compression plates (LCP) have hugely increased in popularity for the treatment of various fracture types. ${ }^{1-3}$ LCPs have proven clinically useful especially in preserving periosteal blood-supply, minimally invasive use, and in poor bone quality (osteoporosis) or bridging-properties in bone-loss. ${ }^{4}$ Currently, locking plates are most frequently applied in distal radius, proximal humerus, distal femur, tibia plateau, proximal and distal tibia, and other peri-articular or periprosthetic fractures. ${ }^{1}$ They are used in order to create stable fixations, allowing for early range of motion exercise. $^{1}$

Research reports on the in vitro biomechanical properties of LCP or comparing two different plate-types for strength and load-to-failure are abundantly available. ${ }^{1}$ However, outcome after clinical use is mainly restricted to case series with one or more fracturetypes without control group. ${ }^{1}$ The number of in vivo studies comparing locking with non-locking plates in a single fracture type is limited. ${ }^{5-9}$ These studies show no definite improvement of outcome or radiographic parameters at final follow-up with the use of locking plates. ${ }^{5-9}$

At our institution, a Level-2 trauma center and teaching hospital, locking plates were introduced and used in ankle fractures since 2006. To date, there are no studies which compare the complication rates in isolated ankle fractures in which the distal fibula fractures were treated with LCP or conventional non-locking plates. As complications might affect outcome and increase costs, the primary aim of the current study was to assess post-operative infection rates of LCP versus one-third tubular (non-locking 
plates) for the treatment of isolated distal fibular fractures. The secondary aims were comparing reduction accuracy and time-to-hardware-removal in both groups. 


\section{Patients and Methods}

This is a retrospective study of all consecutive patients, between January 1, 2004 and December 31, 2009, in which plating of the fibula in a closed ankle fracture was performed. Open ankle fractures $(\mathrm{N}=2)$, fractures treated with lag-screws or syndesmotic screws only, or a transarticular Steinmann-pin $(\mathrm{N}=33)$, and isolated medial malleolar fractures $(\mathrm{N}=16)$ were excluded.

Patient characteristics (age, gender, diabetes, and smoking habits), fracture characteristics (fracture side, Weber fracture type, AO-classification, and number of fractured malleoli), and operation characteristics (delay until operation, duration of surgery, use of tourniquet, plate type, plate length, use of titanium, attending resident or surgeon, and reduction accuracy) were recorded from the patient files, operation reports, and the picture archiving and communication system (PACS). Reduction was classified as anatomical, less than $2 \mathrm{~mm}$ residual lateral displacement of the fibula at the level of the tibial plafond (Mueller nose), or more than $2 \mathrm{~mm}$ residual dislocation of the fibula. This study was performed with approval of the local medical ethics committee.

Infectious wound complications and the need for elective hardware removal were scored., Wound infection was further separated into superficial or minor and deep or major infectious complications by applying the criteria of the Centers for Disease Control and Prevention (CDC) for defining a surgical site infection. ${ }^{10-11}$ Minor complications were defined as a superficial infection treatable with conservative management like oral antibiotics only, without the need for intervention or re-admission. Major complications were defined as a deep infection in need for intervention or readmission, like intravenous antibiotics, removal of hardware, wound debridement with 
or without vacuum assisted closure. The differentiation between superficial and deep infection was made by the surgeon or attending physician. The duration between index operation and removal of hardware and the elective hardware removal percentage was recorded in all patients who had their plate removed.

\section{Operative technique}

All patients received antibiotic prophylaxis according to hospital protocol (third generation cephalosporin). The use of a tourniquet was based on surgeon's preferences. Two different, non-precontoured, plate types were used; first a one-third tubular plate, stainless steel or titanium, with $3.5 \mathrm{~mm}$ screws used as neutralization plate after precontouring, a lag screw was placed for interfragmentary fracture fixation when fracture-type allowed. Secondly, a small fragment (3.5mm) locking (compression) plate, titanium straight metaphyseal or reconstruction type, with angle-stable locking-screws was used (Figure 1). The choice of implant was left at the discretion of the attending physician, as prior to this study no protocol on the usage of locking plates existed. Upon closure, the wound was washed-out with saline. Subcutis was closed using Vicryl ${ }^{\circledR}$ and skin closure was performed using Ethylon ${ }^{\circledR}$. No drains were used.

\section{Data analysis}

The statistical analysis was performed using the Statistical Package for the Social Sciences (SPSS) version 16.0 (SPSS, Chicago, IL). The Kolmogorov-Smirnov test was used to test for normality of the data. The Levene's test was applied to assess homogeneity of variance between data. Since most numeric variables did not show normal distribution or equal variance, all items were regarded as nonparametric for the statistical analysis. A Mann-Whitney U-test (numeric data) or Chi2 analysis (nominal and 
ordinal data) was performed in order to assess statistical significance of difference between the conventional and locking-plate groups. A p-value $<0.05$ was taken as level of statistical significance.Numeric data are expressed as medians with $\mathrm{P}_{25}-\mathrm{P}_{75}$; nominal and ordinal data are shown as numbers with percentages.

Logistic regression models were subsequently developed in order to correct for gender, age, fracture-type, delay to surgery, tourniquet use, plate length, use of titanium, attending resident or surgeon, smoking, and diabetes. A p-value $<0.05$ was taken as level of statistical significance. 


\section{Results}

During the six year study period plating of the distal fibula was performed in 205 patients with an isolated closed ankle fracture. A total of 165 patients received a onethird tubular plate (131 stainless steel and 34 titanium) and 40 patients were treated with a locking compression plate (26 straight metaphyseal and 14 reconstruction). The patient, fracture, and operation characteristics are presented in Table 1.

Both groups had similar patient characteristics. Median age was 48 years. Males and females were equally represented, and both left and right sides were equally affected. One-fifth of patients were smokers, and four percent were diagnosed with diabetes.

The fractures were almost exclusively Weber B and C types (76.1 and 22.4 percent respectively), and mostly unimalleolar $(57.1 \%)$ or bimalleolar $(28.8 \%)$. No statistical difference was found when comparing both treatment groups. The same holds true for AO-classification (data not shown), operation delay (7 days), and operation time (median 60 minutes), the use of a tourniquet (43.9\%), and plate length (median 7 holes). During the study period a total of 10 different surgeons (three trauma surgeons and seven general surgeons) performed the operations. This was similar in both groups. In $66.3 \%$ of cases the patient was operated on by a resident, supervised by one of the staffsurgeons. In $75.1 \%$ of cases, a trauma surgeon led or supervised the operation. In $18.8 \%$ of cases this was done by a general surgeon. This was similar in both groups.

There was a twofold increase in non-anatomical reductions in the locking plate group ( 8.3 versus $15 \%$. This difference was not significant $(\mathrm{p}=0.128)$. 
The wound complication rate for the entire group was 7.8 percent (Table 2). Statistically significantly more wound complications had occurred in the locking plate group $(17.5 \%)$ compared with the conventional plating group $(17.5$ versus $5.5 \% ; \mathrm{p}=0.019)$. This difference was largely due to an 8.3-fold increase in major complications in the locking plate group, for which removal of the plate was necessary $(p=0.008) . \quad$ In order to correct for potential confounding, a multivariable model was developed. The difference in wound complications remained significant in the multivariate analysis, and was not dependent of gender, age, smoking, diabetes, use of titanium, attending resident or surgeon, and Weber classification ( $\mathrm{p}=0.041$; data not shown).

There was no difference between both groups in percentage of hardware removal (overall 27.3\%) at follow-up. With a median of 7.8 months, the time to removal of hardware of locking plates was 2 months sooner than removal of conventional plates, however no statistical significance could be found. 


\section{Discussion}

This is the first report of the comparison of conventional and locking plates for the treatment of isolated distal fibular fractures. A significant increase in wound complications was found in the current study, to the prejudice of the locking plate group. A possible explanation might be the increased thickness of the locking plates $(2.8-3.3$ $\mathrm{mm})$ when compared to the one-third tubular plate $(1.0 \mathrm{~mm})$, even though the screwheads in the one-third tubular plates are more prominent (Figure 2).

Despite a 2-fold higher absolute rate of non-anatomic reductions in the locking-plate group, this was not significantly different from the control group. This could not be attributed to lack of statistical power, which exceeded $90 \%$. Adequate reduction proves more difficult with LCPs compared to conventional non-locking plates. ${ }^{12}$ Jiang et al. identified an increase in malalignment in tibia plateau fractures with the use of locking plates. ${ }^{8}$ This increase might be due to the thickness, and therefore less precontourable nature, of the plate. Reducing fractures 'on the plate' might also prove more difficult with a locking plate.

Plate removal was performed 2 months earlier in the locking-plate group because of prominent hardware or wound complication. The current study unfortunately lacked statistical power to make a definitive statement if this reflects true differences between both groups or not. A post-hoc power-analysis showed that a total of 660 controls and 160 LCPs would be necessary to answer this question with adequate power (i.e., alpha 0.05 , power 0.8 and two-sided testing). Several of these comparative studies showed an increase in hardware irritation. ${ }^{6-8}$ 
New indications in fracture treatment have been sought for fractures which might benefit from locking plates. In a multicentre trial to test the LCP Sommer et al. treated 169 fractures with fourteen different indications. ${ }^{3}$ Fibular fractures represented less than 3 percent in this group. However no complication rate for this group was mentioned. To date there is no literature available to compare the wound complication rate of lockingplates in distal fibular fractures.

Wound complication rates for conventional plating of distal fibular fractures in the literature range from 1.44 percent readmissions for wound complications ${ }^{13}$ up to approximately 15 percent. $^{14-15}$ However most studies report wound complications around 6 percent, which is comparable to the complication rate of the non-locking group in the current study ${ }^{16-17}$ This rate might be slightly higher than for the entire population of surgically treated ankle fractures, because of exclusion of patients treated with lagscrew and syndesmotic-screw only, which have known lower complications rates than plated fractures. ${ }^{18}$ Moreover, the diagnosis wound-infection was made based upon clinical judgement, due to the retrospective nature of this study wound cultures could only occasionally be traced.

The use of a tourniquet was slightly lower in the LCP-group (32 versus 46 percent), which might be explained by the fact that these patients were preferentially operated by a trauma surgeon, whereas the non-LCP-group was operated more frequently by a general surgeon. In the multivariable analysis it was found that operations by the attending surgeon or a resident did not differ significantly in complication rate, even though they may have different soft tissue handling techniques. In a prospective randomised trial, Maffulli et al, found a significantly higher wound complication rate with the use of a tourniquet of 25 percent versus 10 percent without the use of a tourniquet. ${ }^{19}$ Comparable results were identified by Konrad et al. in which a 
difference in wound complications with the use of a tourniquet was detected. In the tourniquet group 4 out of 26 patients experienced a wound complication, versus 2 out of 28 in the non-tourniquet group. ${ }^{20}$

Studies comparing locking and non-locking plates in a single fracture type are scarce. ${ }^{5-9}$ Hahnloser et al. compared dorsally placed locking and non-locking plates in distal radial fractures and showed an overall significant increase in complications associated with the use of locking plates. ${ }^{6}$ Two studies concerning proximal humeral fractures reported similar complication rates in both the locking and non-locking groups. ${ }^{7,9}$ Koshimune et al. did not report on complications in the two different groups. ${ }^{5}$

In order to eliminate the possible influence of titanium versus stainless steel, complication rates in the one-third tubular group, which consisted of 131 stainless steel and 34 titanium non-LCP's, were further investigated. In the stainless steel subgroup 4 minor complications occurred. In the titanium subgroup three minor and 2 major complications were seen. This difference was not statistically different. Difference in complications between the metaphyseal and reconstruction plate were also not seen. In both cases this is probably due to the lack of power. No literature on differences in infection rates between stainless steel or titanium plates could be found.

Even though this study has a retrospective design, and choice of plates was left at the discretion of the attending surgeon, there is currently little data on the use of locking plates in distal fibular fractures. No previous studies on wound complications in distal fibular fractures with the use of locking plates could be found in the literature. One case report was identified in which a non-union occurred after intramedullary nailing of a 
tibia and a concomitant locking plate fixation of a fibular fracture. ${ }^{21}$ The only two studies concerning the use of locking plates in isolated distal fibular fractures that have been published to date are on the biomechanical comparison of locking plates versus conventional plating; both failed to show a greater 'torque to failure' and construct stiffness for the locking plates. $^{22-23}$ 


\section{Conclusion}

The use of locking plates for the treatment of isolated distal fibular fractures is associated with a statistically significant increase in both overall wound complications and major wound complications compared with the use of conventional plating. In light of the current study and findings from the literature, we would caution against the application of the currently used locking compression plates in the treatment of distal fibular fractures. More recently developed low profile locking plates might combine the locking facilities without the bulkiness, which might be the underlying cause of the significant increased infectious complications. These newer plates will also have to undergo thorough clinical investigation. 


\section{References}

1 Anglen J, Kyle RF, Marsh JL, Virkus WW, Watters WC, 3rd, Keith MW, et al. Locking plates for extremity fractures. J Am Acad Orthop Surg 2009;17: 465-72.

2 Wagner M. General principles for the clinical use of the LCP. Injury 2003;34 Suppl 2: B31-42.

3 Sommer C, Gautier E, Muller M, Helfet DL, Wagner M. First clinical results of the Locking Compression Plate (LCP). Injury 2003;34 Suppl 2: B43-54.

4 Schutz M, Sudkamp NP. Revolution in plate osteosynthesis: new internal fixator systems. J Orthop Sci 2003;8: 252-8.

5 Koshimune M, Kamano M, Takamatsu K, Ohashi H. A randomized comparison of locking and non-locking palmar plating for unstable Colles' fractures in the elderly. J Hand Surg Br 2005;30: 499-503.

6 Hahnloser D, Platz A, Amgwerd M, Trentz O. Internal fixation of distal radius fractures with dorsal dislocation: pi-plate or two 1/4 tube plates? A prospective randomized study. J Trauma 1999;47: 760-5.

7 Handschin AE, Cardell M, Contaldo C, Trentz O, Wanner GA. Functional results of angular-stable plate fixation in displaced proximal humeral fractures. Injury 2008;39: 306-13.

8 Jiang R, Luo CF, Wang MC, Yang TY, Zeng BF. A comparative study of Less Invasive Stabilization System (LISS) fixation and two-incision double plating for the treatment of bicondylar tibial plateau fractures. Knee 2008;15: 139-43.

9 Friess DM, Attia A. Locking plate fixation for proximal humerus fractures: a comparison with other fixation techniques. Orthopedics 2008;31:

10 Mangram AJ, Horan TC, Pearson ML, Silver LC, Jarvis WR. Guideline for Prevention of Surgical Site Infection, 1999. Centers for Disease Control and 
Prevention (CDC) Hospital Infection Control Practices Advisory Committee. Am J Infect Control 1999;27: 97-132; quiz 33-4; discussion 96.

11 Petherick ES, Dalton JE, Moore PJ, Cullum N. Methods for identifying surgical wound infection after discharge from hospital: a systematic review. BMC Infect Dis 2006;6: 170 .

12 Smith WR, Ziran BH, Anglen JO, Stahel PF. Locking plates: tips and tricks. J Bone Joint Surg Am 2007;89: 2298-307.

13 SooHoo NF, Krenek L, Eagan MJ, Gurbani B, Ko CY, Zingmond DS.

Complication rates following open reduction and internal fixation of ankle fractures. J Bone Joint Surg Am 2009;91: 1042-9.

14 Tonnesen H, Pedersen A, Jensen MR, Moller A, Madsen JC. Ankle fractures and alcoholism. The influence of alcoholism on morbidity after malleolar fractures. $\mathbf{J}$ Bone Joint Surg Br 1991;73: 511-3.

15 Carragee EJ, Csongradi JJ, Bleck EE. Early complications in the operative treatment of ankle fractures. Influence of delay before operation. J Bone Joint Surg Br 1991;73: 79-82.

16 Konrath G, Karges D, Watson JT, Moed BR, Cramer K. Early versus delayed treatment of severe ankle fractures: a comparison of results. J Orthop Trauma 1995;9: 377-80.

17 Hoiness P, Engebretsen L, Stromsoe K. Soft tissue problems in ankle fractures treated surgically. A prospective study of 154 consecutive closed ankle fractures. Injury 2003;34: 928-31.

18 Tornetta P, 3rd, Creevy W. Lag screw only fixation of the lateral malleolus. J Orthop Trauma 2001;15: 119-21. 
19 Maffulli N, Testa V, Capasso G. Use of a tourniquet in the internal fixation of fractures of the distal part of the fibula. A prospective, randomized trial. J Bone Joint Surg Am 1993;75: 700-3.

20 Konrad G, Markmiller M, Lenich A, Mayr E, Ruter A. Tourniquets may increase postoperative swelling and pain after internal fixation of ankle fractures. Clin Orthop Relat Res 2005189-94.

21 Kokkinakis MC, Bebbington A, Black PRM. Symptomatic fibular non-union after union of accompanying tibial shaft fracture. Injury Extra 2006;37: 135-37.

22 Kim T, Ayturk UM, Haskell A, Miclau T, Puttlitz CM. Fixation of osteoporotic distal fibula fractures: A biomechanical comparison of locking versus conventional plates. J Foot Ankle Surg 2007;46: 2-6.

23 Minihane KP, Lee C, Ahn C, Zhang LQ, Merk BR. Comparison of lateral locking plate and antiglide plate for fixation of distal fibular fractures in osteoporotic bone: a biomechanical study. J Orthop Trauma 2006;20: 562-6. 
Figures and tables

Figure 1. Different plate types used in the current study
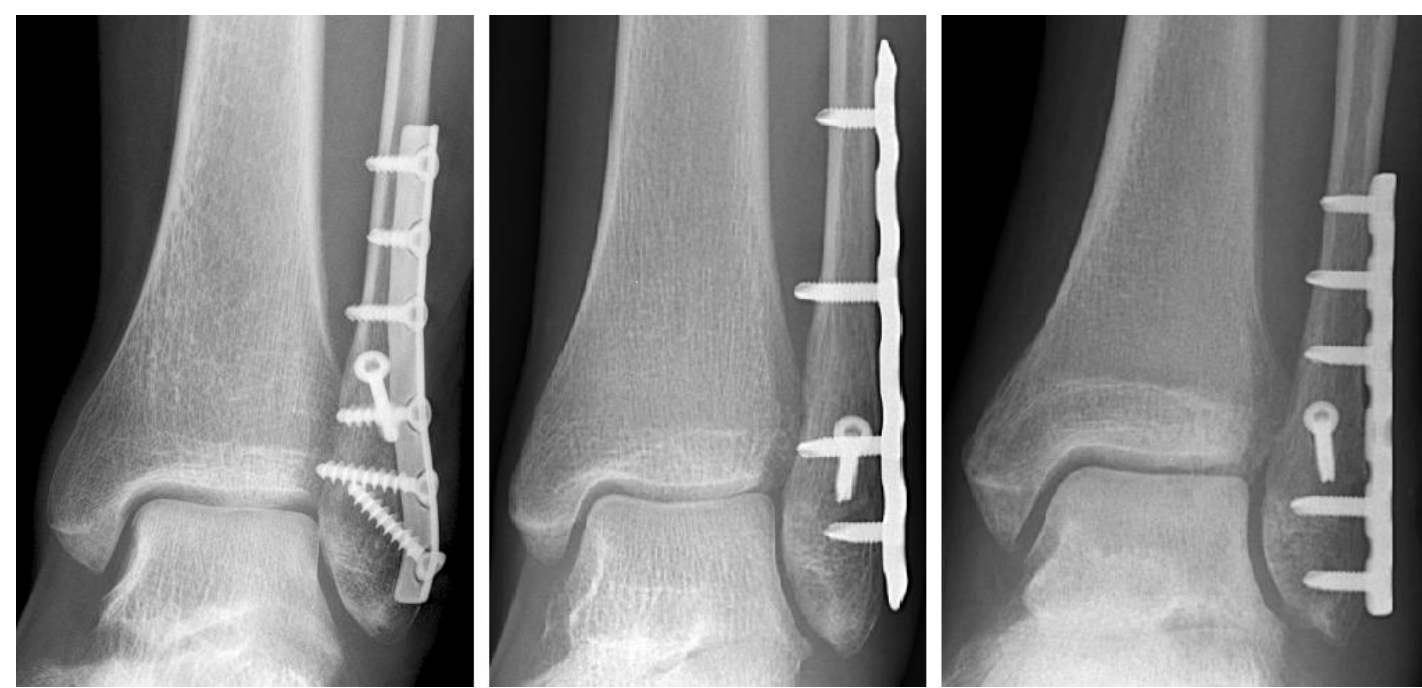

A; Conventional one-third tubular plate, B; straight metaphyseal locking compression plate, C; reconstruction locking plate. 
Figure 2. Profile of different plate types

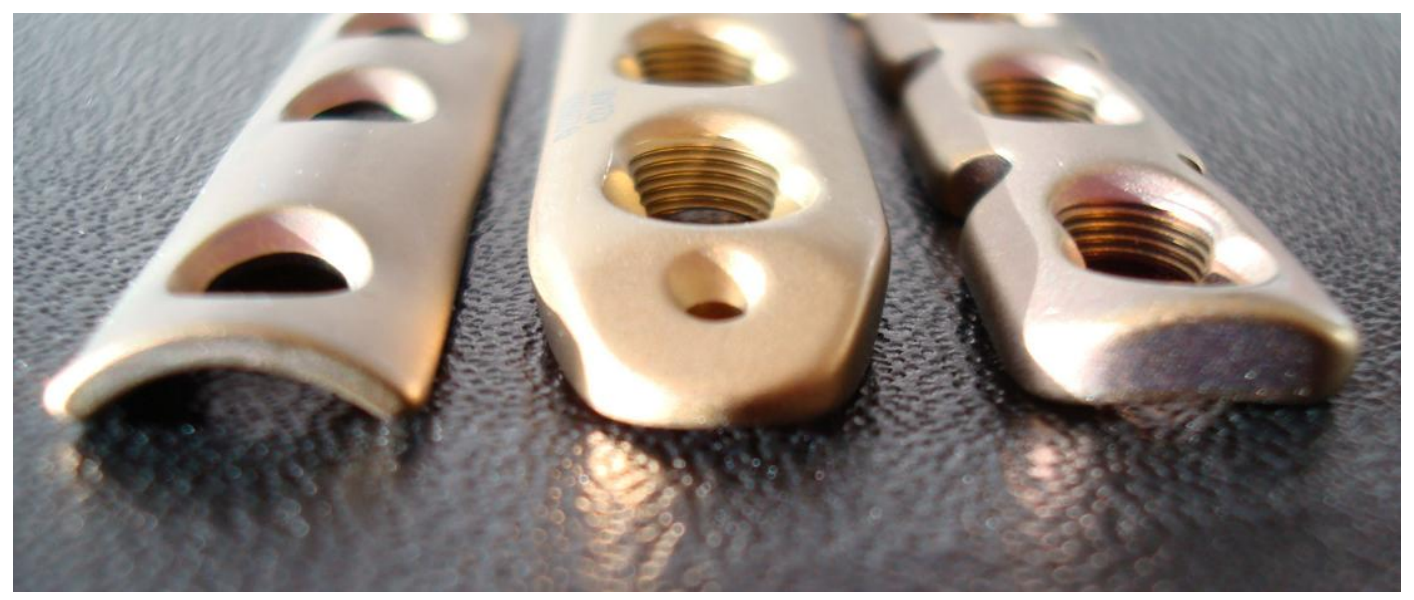

From left to right: Conventional one-third tubular plate, a straight metaphyseal locking compression plate, and the reconstruction locking compression plate. 
Table 1. Patient and fracture characteristics for both patient groups

\begin{tabular}{lcccc}
\hline & $\begin{array}{c}\text { Overall } \\
\text { population }\end{array}$ & $\begin{array}{c}\text { One-third } \\
\text { tubular plate }\end{array}$ & Locking plate & p-value \\
\hline Patients (n) & 205 & 165 & 40 & \\
Male (\%) & $104(50.7)$ & $85(51.5)$ & $19(47.5)$ & $0.725^{\mathrm{a}}$ \\
Age (years) & $48.2(33.5-$ & $48.4(33.5-$ & $46.5(31.6-$ & $0.764^{\mathrm{c}}$ \\
& $61.0)$ & $60.9)$ & $64.7)$ & \\
Smokers (\%) & $44(21.5)$ & $37(22.4)$ & $7(17.5)$ & $0.218^{\mathrm{b}}$ \\
Diabetic (\%) & $9(4.4)$ & $7(4.2)$ & $2(5.0)$ & $1.000^{\mathrm{b}}$ \\
Right sided (\%) & $105(51.2)$ & $85(51.5)$ & $20(50.0)$ & $1.000^{\mathrm{a}}$ \\
Fracture-dislocation (\%) & $26(12.7)$ & $21(12.7)$ & $5(12.5)$ & $1.000^{\mathrm{a}}$ \\
Weber classification (\%) & & & & \\
\multicolumn{1}{c}{ A } & $3(1.5)$ & $3(1.8)$ & $0(0.0)$ & $0.327^{\mathrm{b}}$ \\
$\quad$ B & $156(76.1)$ & $128(77.6)$ & $28(70.0)$ & \\
$\quad$ C & $46(22.4)$ & $34(20.6)$ & $12(30.0)$ & \\
Fracture type (\%) & & & & \\
$\quad$ Unimalleolar & $117(57.1)$ & $96(58.2)$ & $21(52.5)$ & $0.617^{\mathrm{b}}$ \\
$\quad$ Bimalleolar & $57(27.8)$ & $46(27.9)$ & $11(27.5)$ & \\
$\quad$ Trimalleolar & $31(15.1)$ & $23(13.9)$ & $8(20.0)$ & \\
\hline
\end{tabular}

Data is presented as number with the percentage between brackets (categoric data) or as mean with the $\mathrm{P}_{25}$ and $\mathrm{P}_{75}$ between brackets (numeric data). Data were analyzed using the ${ }^{\mathrm{a}}$ Fisher's Exact Test, ${ }^{\mathrm{b}}$ Chi-Square Test, or ${ }^{\mathrm{c}}$ Mann-Whitney U-Test. 
Table 2. Surgical details on both plate groups

\begin{tabular}{lcccc}
\hline & $\begin{array}{c}\text { Overall } \\
\text { population }\end{array}$ & $\begin{array}{c}\text { One-third } \\
\text { tubular plate }\end{array}$ & Locking plate & p-value \\
\hline Operation delay (days) & $7(1-11)$ & $7(1-11)$ & $7.5(1-11)$ & $0.655^{\mathrm{c}}$ \\
Operation time (minutes) & $60(49-76)$ & $60(49-78)$ & $60(52.5-74)$ & $0.686^{\mathrm{c}}$ \\
Tourniquet (\%) & $90(43.9)$ & $77(46.7)$ & $13(32.5)$ & $0.050^{\mathrm{b}}$ \\
Plate length (holes) & $7(6-7)$ & $7(6-7)$ & $7(6-7)$ & $0.588^{\mathrm{c}}$ \\
Operated by resident (\%) & 66.3 & 63.0 & 80.0 & $0.105^{\mathrm{b}}$ \\
\hline Reduction accuracy (\%) & & & & \\
\multicolumn{1}{c}{ Anatomical } & $187(91.2)$ & $153(92.7)$ & $34(85)$ & $0.128^{\mathrm{a}}$ \\
\multicolumn{1}{c}{ Zero to 2mm } & $18(8.8)$ & $12(7.3)$ & $6(15)$ & \\
\multicolumn{1}{c}{ More than 2mm } & $0(0)$ & $0(0)$ & $0(0)$ & \\
\hline
\end{tabular}

Data is presented as number with the percentage between brackets (categoric data) or as mean with the $\mathrm{P}_{25}$ and $\mathrm{P}_{75}$ between brackets (numeric data).

Data were analyzed using the ${ }^{\mathrm{a}}$ Fisher's Exact Test, ${ }^{\mathrm{b}}$ Chi-Square Test, or ${ }^{\mathrm{c}}$ MannWhitney U-Test. 
Table 3. Complication-rates between both patient groups

\begin{tabular}{lcccc}
\hline & $\begin{array}{c}\text { Overall } \\
\text { population } \\
(\mathbf{n = 2 0 5})\end{array}$ & $\begin{array}{c}\text { One-third } \\
\text { tubular } \\
\text { plate }(\mathbf{n}= \\
\mathbf{1 6 5})\end{array}$ & $\begin{array}{c}\text { Locking } \\
\text { plate } \\
(\mathbf{n = 4 0})\end{array}$ & p-value \\
& & $9(5.5)$ & $7(17.5)$ & $0.019^{\mathrm{a}}$ \\
\hline Wound complication & $16(7.8)$ & & & \\
$(\%)$ & & & & \\
Complication type (\%) & & & & \\
$\quad$ Minor & $10(4.9)$ & $7(4.2)$ & $4(10)$ & \\
$\quad$ Major & $6(2.9)$ & $2(1.2)$ & $11(27.5)$ & $1.000^{\mathrm{a}}$ \\
Plate removal (\%) & $56(27.3)$ & $45(27.3)$ & $0.117^{\mathrm{c}}$ \\
Plate removal (months) & $8.8(5.2-13.7)$ & $10(5.6-14.0)$ & $7.8(5.0-8.8)$ & \\
\hline
\end{tabular}

Data is presented as number with the percentage between brackets (categoric data) or as mean with the $\mathrm{P}_{25}$ and $\mathrm{P}_{75}$ between brackets (numeric data).

Data were analyzed using the ${ }^{\mathrm{a}}$ Fisher's Exact Test, ${ }^{\mathrm{b}}$ Chi-Square Test, or ${ }^{\mathrm{c}}$ MannWhitney U-Test. 